\title{
Intuition und Erkenntnis: Philosophische Positionen der Neuzeit
}

Als Auftakt zur Ergründung des Intuitionsbegriffs und des epistemischen Status der Intuitionen sollen einige bedeutende Intuitionsverständnisse der Neuzeit dargestellt werden. Die Wahl fällt auf Descartes, Spinoza, Locke, Kant und Goethe. Diese Auswahl ergibt sich aus einem systematisch ausgerichteten Erkenntnisinteresse. Demzufolge werden Stationen dargestellt, die für ein heute adäquates Intuitionsverständnis und für eine Beantwortung der Frage nach dem Beitrag der Intuition zur Erkenntnis besonders relevant sind. Zudem finden sich interessante Bezüge zwischen den dargestellten Positionen. Eine genauere Begründung der Auswahl soll zugleich als Vorschau dienen:

1. Bei Descartes findet sich erstmals eine ausführliche Behandlung der Intuition als Erkenntnisgattung, die nicht an starke metaphysische Voraussetzungen gebunden ist. Durch die damit verbundene klare Fokussierung auf das epistemische Subjekt stehen bei Descartes Fragen im Zentrum, die für die zeitgenössische Auseinandersetzung mit der Intuition von großer Relevanz sind; insbesondere die folgenden: Wie ist es, eine Intuition zu haben? Welcher Zusammenhang besteht zwischen Intuition und Rechtfertigung bzw. Wissen, welcher zwischen Intuition und Schließen?

2. Spinoza und Locke greifen Cartesische Gedanken zur Intuition auf und erweitern oder modifizieren diese auf interessante Weise. Spinozas Intuitionskonzeption wird in Bezug auf die postulierte Möglichkeit des intuitiven Zugangs zu Wesensbegriffen wichtig. Mittels Intuition würde demnach der unmittelbare Zugang zum Wesen der Dinge ermöglicht; ein großes epistemologisches Versprechen, dessen Berechtigung zu prüfen sein wird.

3. Kants Bedeutung für jede ernsthafte Auseinandersetzung mit der Intuition und deren epistemischem Status darf nicht übersehen werden. Seine Skepsis in Bezug auf eine Intuition, die mehr wäre als empirische Anschauung lohnt sich aus mindestens zwei Gründen nachzuvollziehen: Einerseits lässt sich Kant folgend näher bestimmen, was eine genuine Intuition ausmachen würde und was sie leisten müsste. Andererseits wird Kants Verständnis der Imagination in Bezug auf das Verhältnis zwischen Intuition und mentalen Modellen erhellend sein. Um diesen neuen Weg 
darstellen zu können, müssen Kants Grundüberlegungen zur Intuition bekannt sein.

4. Goethe nimmt in seinen Pflanzenstudien Spinozas Arbeiten zur Intuition auf und wertet damit die Intuition nach Kant wieder auf. Er geht allerdings nicht mehr den rationalistischen Weg Descartes' und Spinozas, sondern verbindet die intuitive Erkenntnis mit der empirischen. Für die Darstellung der Arbeiten Goethes zur Intuition sowie der Verbindungen zu Spinoza und Kant ist Eckart Försters Abhandlung Die 25 Jahre der Philosophie eine hilfreiche Grundlage. ${ }^{12}$

Mit diesen Überlegungen soll nicht gesagt sein, dass nicht auch andere Positionen - wie etwa die erwähnte phänomenologische - für die vorliegende Studie bereichernd sein könnten. Die hier getroffene Auswahl reicht aber aus, um die weiteren Erörterungen zum Intuitionsbegriff auf ein solides Fundament zu stellen, was in der aktuellen Intuitionsdiskussion oft gänzlich unterlassen wird. Weil sich frühe Denker der neuzeitlichen Philosophie bereits intensiv mit der Intuition als Erkenntnisart auseinandergesetzt haben, lohnt es sich, ihre Studien zur Kenntnis zu nehmen um erste Hinweise über die Intuition und deren epistemischen Status zu erhalten. Diese Hinweise werden als Referenzpunkte für die weitere Arbeit dienen.

\subsection{Descartes: Intuition und Deduktion als zwei Hauptwege der Erkenntnis}

Im philosophischen Werk René Descartes' (1596-1650) spielt der Intuitionsbegriff eine zentrale Rolle; dies bereits in den Regulae ad directionem ingenii (>Regeln zur Ausrichtung der Geisteskraft $<$ ). ${ }^{13}$ Die zentrale Textpassage zum Intuitionsbegriff in den Regulae ist die Folgende:

Unter Intuition verstehe ich nicht das Vertrauen in die unbeständigen Sinne oder das trügerische Urteil einer schlecht zusammensetzenden Anschauung, sondern einen so einfachen und deutlichen Begriff des reinen und aufmerksamen Geistes, daß über das, was wir einsehen, schlichtweg kein Zweifel mehr übrigbleibt. Oder, was dasselbe ist: einen zweifelsfreien Begriff des reinen und aufmerksamen Geistes, der allein im Licht der Vernunft seine Wurzel hat und deshalb sogar gewisser ist als die Deduktion selbst, weil er einfacher ist als sie,

\footnotetext{
12 Vgl. Förster (2011).

13 Aus den Regulae wird mit den Band- und Seitenangaben der Werkausgabe von Adam und Tannery zitiert (Kürzel AT X, gefolgt von der Seitenangabe). Die deutschen Übersetzungen folgen der Meiner-Ausgabe, übers. von Christian Wohlers (2011).
} 
die ihrerseits vom Menschen auch nicht verkehrt durchgeführt werden kann $[\ldots] \cdot{ }^{14}$

Descartes zufolge weist die Intuition also zwei Hauptmerkmale auf: Erstens ist sie dem »reinen Geist« zugeordnet und damit - ganz im Sinne des Rationalismus - von der sinnlichen Wahrnehmung klar zu unterscheiden. Zweitens geht sie mit Gewissheit einher: Was intuitiv erkannt wird, wird zunächst zweifelsfrei erkannt. ${ }^{15}$

Aufgrund der Gewissheit, die Descartes der intuitiven Erkenntnis zuschreibt, spielt diese im Rahmen der Methodenlehre der Regulae eine Schlüsselrolle. Der Kern dieser Methodenlehre kommt in der 5. Regel prägnant zum Ausdruck:

Die gesamte Methode besteht in der Ordnung und Gliederung dessen, worauf die Schärfe des Geistes zu richten ist, um eine Wahrheit herauszufinden. Diese Methode verfolgen wir dann exakt, wenn wir verwickelte und dunkle Propositionen stufenweise auf einfachere zurückführen und danach versuchen, von der Intuition der allereinfachsten über dieselben Stufen zur Erkenntnis aller anderen aufzusteigen. ${ }^{16}$

Die »allereinfachsten« Propositionen werden intuitiv erfasst, wogegen die Denkschritte, welche diese Ausgangspropositionen mit allen weiteren Propositionen verbinden, als deduktive Schlüsse bezeichnet werden. ${ }^{17}$ Jede Proposition darf nur dann als wahr erachtet werden, wenn sie mittels einer ununterbrochenen Kette selbstevidenter Erkenntnisschritte aus den Ausgangspropositionen herleitbar ist. ${ }^{18}$

Nun kann nicht nur eine Ausgangsproposition, sondern auch das direkte Folgen einer Proposition aus einer anderen intuitiv erkannt werden (intuitiver Schluss). Descartes verdeutlicht dies anhand eines mathematischen Beispiels: $>2+2=4$ < ist selbstevident, d.h., die Richtigkeit dieser Gleichung ist intuitiv erkennbar. Dasselbe gilt für $>3+1=4$ <. Ebenso selbstevident und damit intuitiv erkennbar ist die Wahrheit der Proposition $>2+2=3+1<$, da sie direkt aus den zuvor genannten Propositionen hervorgeht. ${ }^{19}$ Auch Descartes' berühmter

\footnotetext{
$14 \quad \mathrm{AT} \mathrm{X}_{3} 68$.

15 Hiermit reiht sich Descartes in die vorscholastische Tradition ein, welche die Intuition im Bereich des Intellekts (im Gegensatz zum Bereich der Wahrnehmung) verortet (vgl. Beck, 1952, S. 53; Kobusch, 1976, S. 524-528; Perler, 2006, S. 53).

$16 \quad$ AT X $_{381 .}$

17 Eine Ausnahme bilden die intuitiven Schlüsse, die von einer intuitiv erkannten Ausgangsproposition ausgehen können; siehe weiter unten zu >ich denke, ich existiere<.

18 Vgl. Beck (1952, S. 47).

19 Vgl. AT X 369.
} 
Schluss vom eigenen Denken auf die eigene Existenz ist in der ursprünglichen Fassung der Meditationes als intuitiver Schluss zu verstehen. Die Wahrheit des $>$ ich denke < ist unmittelbar einleuchtend und selbstevident; >ich denke < ist also intuitiv erkennbar. Descartes zufolge lässt sich aus dieser Proposition ebenfalls intuitiv auf >ich existiere< schließen. ${ }^{20} \mathrm{Im}$ Gegensatz dazu suggeriert die von Descartes im Discours verwendete Formel >ich denke, also bin ich einen unvollständigen Syllogismus. Um ihn zu vervollständigen, müsste die Prämisse salles Denkende existiert < eingeschoben werden, wodurch sich die Konklusion >ich existiere aus den beiden genannten Prämissen deduktiv ableiten ließe. Doch diese Auffassung des Cartesischen Gedankens ist - zumindest in Bezug auf seine in den Meditationes dargestellte Form - falsch, da das in der zweiten Prämisse formulierte Wissen nicht benötigt wird, um von >ich denke< auf >ich existiere zu schließen. Vielmehr ist dieser Schluss unmittelbar und selbstevident, d.h. intuitiv. ${ }^{21}$

Mehr als Intuition und Deduktion braucht es nicht, um Wissen zu erlangen, wie Descartes betont. ${ }^{22}$ Dies liegt daran, dass Intuition und Deduktion komplementär zueinander wichtige Rollen im Erkenntnisprozess einnehmen: Durch Intuition nimmt das Erkenntnissubjekt die Anfangsgründe ganzheitlich und augenblicklich wahr. Descartes führt Beispiele hierfür an:

So kann jeder kraft seines Gemüts intuitiv erkennen, daß er existiert, daß er denkt, daß ein Dreieck nur von drei Linien und eine Kugel nur von einer Oberfläche begrenzt wird und dergleichen, von dem es sehr viel mehr gibt als die meisten Leute bemerken, weil sie es ablehnen, ihr Denken so leichten Dingen zuzuwenden. $^{23}$

Die intuitive Erkennbarkeit dieser Propositionen macht sie zu den selbstevidenten Anfangsgründen, auf die mittels zuverlässiger Deduktion sämtliches Wissen sicher aufgebaut werden kann. Die Deduktion ist dabei als »eine Bewegung bzw. eine bestimmte Abfolge« zu verstehen, bei der im Unterschied zur Intuition "gegenwärtige Evidenz nicht notwendig « ist, da sie »ihre Gewissheit vielmehr gewissermaßen dem Gedächtnis entnimmt $\ll^{24}$

\footnotetext{
20 Hier wird der Propositionalismus in Bezug auf Intuition noch nicht in Frage gestellt, da Descartes auch im lateinischen Originaltext den Ausdruck propositiones verwendet (siehe etwa die oben zitierte Einleitung zur 5 . Regel, AT X 381 ).

21 Descartes betont dies in den Zweiten Erwiderungen zu den Meditationes (vgl. AT VII 140; Markie, 2008, S. 145f.).

22 Vgl. AT X 372; Ariew et al. (2003, S. 143); Perler (2006, S. 52).

$23 \quad$ AT X 368.

$24 \quad$ AT X 370.
} 
Auch wenn die deduktive Lesart des >ich denke, ich existiere nicht Descartes' ursprünglicher Intention entspricht, zeigt sich anhand dieses Beispiels, dass sich eine Klasse von Schlüssen sowohl intuitiv als auch deduktiv vollziehen lässt. Descartes bestätigt in den Regulae:

daß zwar jene Propositionen, die aus ersten Prinzipien unmittelbar geschlossen werden, je nach der Verschiedenheit des Gesichtspunkts entweder durch Intuition oder durch Deduktion erkannt werden, die ersten Prinzipien selbst aber nur durch Intuition, die entfernten Schlüsse hingegen allein durch Deduktion. ${ }^{25}$

Auf die erwähnten Beispiele bezogen bedeutet dies, dass eine Person den Schluss von $>2+2=4$ < und $>3+1=4<\mathrm{zu}>2+2=3+1<$ intuitiv oder deduktiv mittels eines einfachen mathematischen Beweises - vornehmen kann. Ebenso kann sie aus dem unmittelbar einleuchtenden sich denke< intuitiv auf sich existiere< schließen oder vom selben Anfangsgrund aus deduktiv, d.h. mittels vollständigem Syllogismus, zur Konklusion >ich existiere kommen. Unmöglich ist hingegen einerseits, dass die Anfangsgründe des Wissens (z.B. sich existiere $<$ deduktiv und andererseits, dass die weiter entfernten Schlussfolgerungen intuitiv festgestellt werden.

Descartes weist der Intuition eine zentrale Bedeutung für die menschliche Erkenntnis zu, da sie infallibles Wissen zu vermitteln und auf diese Weise den Zugang zu einem Wissensfundament zu garantieren scheint. Doch verweist die Gewissheit der Intuition wirklich auf mehr als auf ein subjektives Erleben der Person (psychologische Dimension), hat sie eine objektive Entsprechung (logische Dimension)? ${ }^{26}$ In Bezug auf diese Frage scheint Descartes im Laufe der Zeit skeptischer geworden zu sein. Während etwa mathematische Intuitionen wie $>2+3=5$ < in den Regulae noch als Paradebeispiel für selbstevidente Anfangsgründe des Wissens dienen, können sie gemäß Meditationes durch einen Betrügergott eingeflößt werden und daher falsch sein. Diese Befürchtung kann Descartes erst durch mehrere explizite Denkschritte - also keineswegs intuitiv - widerlegen. Dies scheint auf eine Ebene der Wahrheit hinzuweisen, die uns nicht intuitiv zugänglich ist. Dasselbe lässt sich anhand des >ich denke, ich existiere zeigen. In den Regulae erscheint dies, ebenso wie $>2+3=5$, als intuitiv erkennbar und somit gewiss. Dagegen ist die Pointe des später im Discours und in den Meditationes entwickelten Gedankengangs, dass erst aus der Möglichkeit der Täuschung auf das Vorhandensein eines sich täuschenden und somit denkenden Dings geschlossen werden kann. Dies

$25 \quad$ AT X 370; vgl. Gerten (2001, S. 141).

26 Vgl. Beck (1952, S. 65). 
ist offenbar eine deduktiv ermittelte Gewissheit, auch wenn sich denke, ich existiere $<$ bereits intuitiv als gewiss erschienen war.

Aufgrund der bisherigen Betrachtungen scheint es sinnvoll, zwischen Zweifel erster und zweiter Stufe zu unterscheiden: Auf der ersten Stufe, d.h. psychologisch, scheint Selbstevidentes unbezweifelbar, auf der zweiten Stufe kann ein Subjekt jedoch immer an der Zuverlässigkeit seiner Erkenntnis, insbesondere auch seiner Intuition, zweifeln: Bin ich so disponiert, dass ich die richtigen Intuitionen habe, oder werden mir, etwa durch einen Betrügergott, falsche Intuitionen eingeflößt?27 Die Gründe für die Ausräumung dieser Zweifel müssen zusätzlich, d.h. deduktiv, geliefert werden; es geht bei dem späteren Cartesischen cogito-Argument nicht mehr um eine Intuition im engeren Sinne. Unmittelbarkeit und Gewissheit sind somit nur noch an die Perspektive des Erkenntnissubjekts gebundene Merkmale der Intuition. Sie halten dem Zweifel erster Stufe (etwa im Unterschied zu Vermutungen oder Bauchgefühlen), aber nicht zwingend dem Zweifel zweiter Stufe stand. Damit ist bereits bei Descartes die Neuorientierung der Merkmale Unmittelbarkeit und Gewissheit, weg von einer objektiv-epistemologischen Bedeutung (als Eigenschaft, die infallible Erkenntnis ermöglicht) und hin zu einer subjektiv-phänomenalen Bedeutung (wie es für ein Subjekt ist, eine Intuition zu haben) angelegt. Auf diese Unterscheidung wird später (Kap. 3.1) zurückzukommen sein.

Zusammenfassend lässt sich hinsichtlich des Zusammenhangs zwischen Intuition und Erkenntnis bei Descartes feststellen, dass Intuition einen privilegierten Zugang zu Wissen ermöglicht, womit eine Diskrepanz zwischen dem Cartesischen und dem heute verbreiteten Intuitionsverständnis bemerkbar wird: hier ein >Bauchgefühl $<$ und etwas Diffuses, dort ein hochrationaler Königsweg der Erkenntnis, der Zugang zu den Anfangsgründen unseres Wissens gewähren kann. Das Cartesische Intuitionsverständnis wurzelt in einer rationalistischen Erkenntnistheorie, die das intuitive Wissen als apriorisches Wissen behandelt. Diese Intuitionskonzeption hat eine bedeutende Stellung in der philosophischen Diskussion nach Descartes behalten und ist wohl der Hauptgrund für die starke Diskrepanz zwischen dem philosophischen und dem heute verbreiteten Intuitionsverständnis. Zugleich nimmt Descartes auf der phänomenalen Ebene Bestimmungen der Intuition vor, welche über das rationalistische Verständnis hinaus Gültigkeit haben und an welche sich später anknüpfen lässt. 
Im philosophischen Werk Baruch de Spinozas (1632-1677) nimmt die Intuition als Erkenntnisgattung einen zentralen Stellenwert ein. Dabei kann Spinozas Arbeit in diesem Bereich treffend als »kritische Überbietung cartesianischer Einsichten «bezeichnet werden. ${ }^{28}$ Das Cartesische Material wird durch Spinoza differenziert, modifiziert und in die komplexe Ontologie und Epistemologie seines Hauptwerks Ethica (vollendet 1675) eingebettet.

Spinoza unterscheidet gemäß der Art, wie wir Universalbegriffe bilden, zwischen drei Erkenntnisgattungen: Meinung oder Vorstellung (opinio, imaginatio), Vernunft (ratio) und intuitive Erkenntnis (scientia intuitiva). ${ }^{29}$ Unter opinio und imaginatio subsumiert Spinoza zweierlei: Zum einen gehört die aus der Sinneswahrnehmung stammende Erfahrung und Art der Begriffsbildung (experientia vaga) dazu. Diese stellt dem Verstand die Einzeldinge »verstümmelt [...], verworren und ohne Ordnung « dar. ${ }^{30}$ Zum anderen gehört $\mathrm{zu}$ opinio und imaginatio die Begriffsbildung, die sich durch Erinnerungen und Assoziationen beim Erfassen von bereits vorhandenen Zeichen (wie z.B. dem Hören und Lesen von Worten) einstellt. Wie sich durch die angeführte Wortwahl bereits andeutet, stellt sich Spinoza mit der skeptischen Betrachtung der Erkenntnis aus direkter oder durch Zeichen vermittelter Sinneswahrnehmung in die Cartesianisch-rationalistische Tradition. ${ }^{31}$ Die Erkenntnis der zweiten Gattung, die ratio, bildet ebenfalls Universalbegriffe, jedoch nun verstanden als Gemeinbegriffe (notiones communes). Im Gegensatz zu der durch Sinneswahrnehmung und Vorstellung vermittelten experientia vaga vermag diese Erkenntnis »adäquate Ideen« zu vermitteln. Die dritte Erkenntnisgattung bezeichnet Spinoza als scientia intuitiva. Auch diese führt zu adäquaten Ideen: »Und diese Gattung des Erkennens schreitet von der adäquaten Idee dessen, was die Essenz gewisser Attribute Gottes ausmacht, weiter zu der adäquaten Erkenntnis der Essenz von Dingen «. ${ }^{32}$

Unter >adäquate Idee< versteht Spinoza »eine Idee, die, insofern sie in sich selbst und ohne Beziehung auf einen Gegenstand betrachtet wird, alle Eigenschaften oder inneren Merkmale einer wahren Idee hat «. ${ }^{33} \mathrm{Im}$ Unterschied

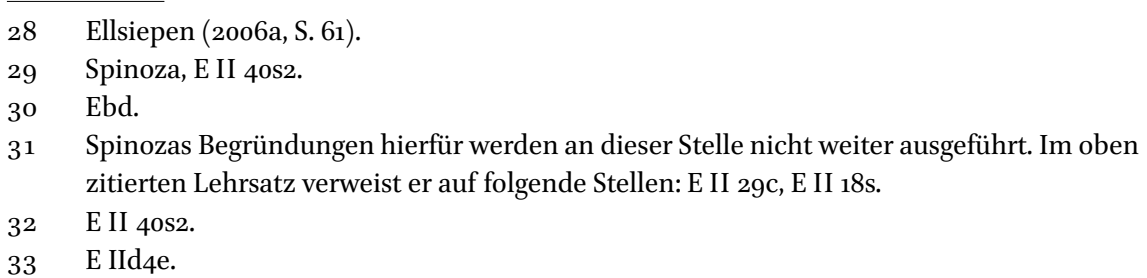


zu >Wahrheit< bezieht sich >Adäquatheitく auf die inneren Eigenschaften der wahren Idee. Diese allein sind dem Erkenntnissubjekt zugänglich, weshalb sich ihm wahre Ideen als adäquate Ideen anzeigen. Der Gedanke, dass ausschließlich ratio und scientia intuitiva adäquate Ideen vermitteln, erweist die rationalistische Verwandtschaft zwischen Descartes und Spinoza. Die beiden Erkenntnisgattungen entsprechen den Cartesischen Erkenntniswegen der Deduktion und Intuition, während Descartes wie auch Spinoza Sinneswahrnehmungen im Bereich des Unsicheren verorten.

Zwar liefern sowohl ratio als auch scientia intuitiva adäquate Ideen, aber nur mit letzterer gelangt das Erkenntnissubjekt zum Wesen der Dinge. Dies erläutert Spinoza am Beispiel eines Kaufmanns:

Es sind drei Zahlen gegeben, zu denen man eine vierte finden möge, die sich zu der dritten verhält wie die zweite zu der ersten. Kaufleute multiplizieren ohne Zögern die zweite mit der dritten und dividieren das Produkt durch die erste, und dies, weil sie entweder noch nicht vergessen haben, was sie von ihren Lehrern ohne irgendeinen Beweis gehört haben, oder weil sie es oft bei ganz einfachen Zahlen herausgefunden haben, oder endlich kraft des Beweises von Lehrsatz $19 \mathrm{im}$ 7. Buch des Euklid, nämlich aus der gemeinsamen Eigenschaft von Proportionalzahlen. Bei ganz einfachen Zahlen ist freilich nichts davon erforderlich. Sind z. B. die Zahlen 1, 2 und 3 gegeben, gibt es niemanden, der nicht sieht, dass 6 die vierte Proportionalzahl ist, und das sehen wir viel klarer, weil wir gerade diese Zahl, die vierte, allein aus dem Verhältnis der ersten zur zweiten Zahl, das wir mit einem Blick sehen, erschließen. ${ }^{34}$

Der Kaufmann geht zunächst den Weg der opinio und imaginatio, indem er sich entweder auf etwas vom Lehrer Gehörtes (und nicht eigenständig Ergründetes) oder auf eine Erinnerung stützt, wobei er aufgrund der Erfahrung annimmt, dass sich dieses Wissen auch im vorliegenden Fall bewähren wird. Es handelt sich hier also um unsichere Meinungen und Vorstellungen, die keine adäquate Erkenntnis vermitteln können. Anders ist der Fall gelagert, wenn der Kaufmann den mathematischen Sachverhalt durchschaut und zur Anwendung bringt, also den Lehrsatz Euklids. Hier zeigt sich die zweite Erkenntnisgattung (ratio), die hierdurch vermittelte Erkenntnis ist adäquat. Ebenfalls adäquat ist die vermittelte Erkenntnis im dritten möglichen Fall: hier erfasst der Kaufmann »auf einen Blick« (uno intuitu), dass es sich bei der nächsten Proportionszahl um 6 handeln muss. In solchen Fällen sagen wir oft, dass wir die Lösung »sofort sehen« und nicht einen mathematischen 
Denkweg beschreiten müssen, wie dies bei der zweiten Erkenntnisgattung der Fall wäre. ${ }^{35,36}$

Im Kaufmann-Beispiel sind die bei Descartes festgehaltenen Kennzeichen einer intuitiven Erkenntnis, Unmittelbarkeit und Gewissheit, wiederum gegeben. Jedoch scheint das Verb, welches Spinoza im Zusammenhang mit dem Kaufmann-Beispiel wählt nicht zu passen: Die Intuition schreite von Attributen Gottes zum Wesen der Dinge fort (procedit). ${ }^{37}$ Wie kann es bei der Intuition ein Fortschreiten geben, wenn diese doch unmittelbar ist? Das Verb procedere markiert Diskursivität und scheint damit vielmehr zur ratio als zur scientia intuitiva zu gehören. Spinoza wählt das Verb procedere nicht unbedacht; Deduktion und somit Diskursivität muss notwendigerweise zur scientia intuitiva gehören. ${ }^{38}$ Der Grund dafür ist, dass die Objekte, auf die sich unsere Erkenntnis richten kann, in Spinozas Ontologie allesamt Modi der einen göttlichen Substanz sind. ${ }^{39}$ Modi sind Arten, in denen die eine Substanz in der Welt gegeben ist (wie z.B. mein Geist, mein Körper). Diese Modi können nur als aus einem der unendlichen Attribute der einen Substanz (z.B. Ausdehnung) folgend begriffen werden. Nähmen wir die Erkenntnisgegenstände nicht als Modi, sondern als Einzeldinge, so können wir uns nur mittels experientia vaga auf diese beziehen. Jedoch scheint aufgrund dieser Überlegung die scientia intuitiva nicht von Unmittelbarkeit, sondern von Diskursivität gekennzeichnet zu sein, was der Aussage des Kaufmann-Beispiels widersprechen würde.

Ob dieser Widerspruch tatsächlich besteht, ist allerdings zweifelhaft. Nicht zu vernachlässigen ist der Unterschied zwischen der Reflexion über Erkenntnis, wie sie Spinoza in seinem Werk vornimmt, und der Erkenntnis an sich. Auch wenn wir mit Spinoza auf der reflexiven Ebene erkennen mögen, dass die Modi aus den Substanzen via Attribute folgen, so impliziert dies nicht, dass wir im Erkenntnismoment eine Folgerung vornehmen und somit diskursiv denken würden. Vielmehr können die auf der reflexiv-philosophischen Ebene stattfindenden Folgerungen - mit der Konzeption der Adäquatheit im Zentrum - erklären, warum die intuitive Erkenntnis unmittelbar und gewiss ist.

35 Vgl. van Zantwijk (2011, S. 80).

36 Wenn es um eine genaue Lesart Spinozas geht, muss kritisch untersucht werden, ob dieses Beispiel eine genuine Anwendung der Intuition oder vielmehr eine Analogie zur derselben ist. Zahlen sind nach Spinoza eine Form von Zeichen und müssen als solche im Bereich der ratio verbleiben, womit die Rechnung des Kaufmanns, wie jede Zahlenoperation, niemals vollständig intuitiv sein kann (vgl. Ellsiepen 2006b, S. 147).

37 Vgl. E II 40 os2.

38 Vgl. Schmidt $(1975$, S. 63$)$.

39 Vgl. E I 15. 
Selbst wenn wir die Nicht-Diskursivität der scientia intuitiva anerkennen, bleibt aber die Frage offen, ob diese Einzigartiges leistet oder ob der Mensch auf sie verzichten und alles gleichermaßen mittels ratio, d.h. durch diskursives Denken, erkennen könnte. Diese Frage hätte bereits bei Descartes gestellt werden können; Spinoza hat nun eine interessante Antwort darauf. Zur Darstellung dieser Antwort muss genauer betrachtet werden, welches die Produkte der ratio bzw. der scientia intuitiva sind, zu denen wir durch den jeweiligen Erkenntnisprozess kommen. Laut Spinoza handelt es sich bei diesen Produkten um Begriffe, die wir bilden. Im Allgemeinen nennt Spinoza diese Begriffe Universalbegriffe (notiones universales). ${ }^{40}$ Universalbegriffe entstehen durch alle drei Erkenntnisgattungen, auch durch die unzureichende Erkenntnis erster Gattung. Davon zu unterscheiden sind die Gemeinbegriffe (notiones communes), eine spezifische Form von Universalbegriffen, die auf rational-deduktivem Weg gebildet werden. Diese »gehen vom Allgemeinen zum Einzelnen, geben Auskunft über unsere Vorstellungweisen «. ${ }^{41}$ Eine weitere Form von Universalbegriffen sind die Wesensbegriffe (notiones essentiae rerum), die auf dem Weg der scientia intuitiva zustande kommen. Im Unterschied zu den Gemeinbegriffen gehen die Wesensbegriffe »vom Einzelnen zum Allgemeinen, erfassen das Wesen der Einzeldinge «. ${ }^{42}$

Spinozas Ausführungen beinhalten also die epistemisch zweifellos bedeutsame Behauptung, dass wir erst mittels der scientia intuitiva das Wesen der Dinge (essentiae rerum) vollständig erfassen können. Die Wesensbegriffe erlauben dem Erkenntnissubjekt einen tieferen Einblick in die Realität als dies bei den Gemeinbegriffen der ratio und noch mehr bei den Begriffen der experientia vaga der Fall ist. Hauptgrund dafür ist in Spinozas Ontologie die direkte Verbindung mit Gott, die via Wesensbegriffe der scientia intuitiva hergestellt ist und diese zur höchsten Erkenntnisgattung macht: »Je mehr wir Einzeldinge einsehen, desto mehr sehen wir Gott ein«, denn »Gott ist nicht nur die bewirkende Ursache der Existenz von Dingen, sondern auch die ihrer Essenz «. ${ }^{43}$ Das Wesen der Dinge zu erfassen, heißt somit für Spinoza, die Welt zugleich unter dem Gesichtspunkt des Göttlichen, Ewigen zu begreifen (sub

\footnotetext{
40 Vgl. E II 40 os2.

41 Wild (2012, S. 4).

42 Ebd. - Spinoza verwendet den Terminus notiones essentiae rerum nicht. Aus der weiter oben angeführten Definition der scientia intuitiva (»schreitet ... fort zur adäquaten Erkenntnis des Wesens der Dinge«) darf er in Analogie zu den beiden anderen erwähnten Begriffsarten gebildet werden, um das Spezifische der dritten Erkenntnisgattung zu akzentuieren.

E V 24; E I 25.
} 
specie aeternitatis). ${ }^{4}$ Jedoch bleibt offen, wie mit der skizzierten Methode der scientia intuitiva das Wesen der Einzeldinge erfasst werden kann, zumal wir die spezifischen Eigenschaften der Einzeldinge nicht »aus der formalen Wesenheit der Attribute Gottes ableiten können«, wie Eckart Förster zu Recht geltend macht: »alle Dinge, lebendige wie unbelebte, hätten sonst das gleiche Wesen! ${ }^{45}$

Trotz offener Fragen wie dieser, die mit Spinozas voraussetzungsreicher Ontologie zusammenhängen, sind die zentralen Aspekte seiner Intuitionsauffassung für die weitere Untersuchung bedeutsam. Insbesondere wird auf Spinozas zur Abhebung der Intuition getroffene Unterscheidung zwischen Gemein- und Wesensbegriffen zurückzukommen sein.

\subsection{Locke: Intuition als unmittelbarste Erkenntnis}

John Locke (1632-1704) setzt sich in seinem erkenntnistheoretischen Hauptwerk An Essay concerning Human Understanding (1690) zum Ziel, Ursprung, Gewissheit und Ausdehnung unserer Erkenntnis zu erforschen. ${ }^{46}$ Mit den in diesem Werk verteidigten empiristischen Kernthesen, wonach alle unsere Ideen aus der Erfahrung stammen und die Erfahrung der alleinige Prüfstein für die Geltung von Aussagen sei, stellt er sich gegen Vertreter des Rationalismus wie Descartes und Spinoza. Trotz dieser Opposition bestehen deutlich erkennbare Parallelen zwischen Lockes Intuitionskonzeption und derjenigen des Rationalisten Descartes. So behandelt Locke im Essay ebenfalls die Intuition und baut hierbei auf dem Cartesischen Intuitionsbegriff auf. ${ }^{47}$

Einleitend im Essay definiert Locke Erkenntnis als »die Wahrnehmung des Zusammenhangs und der Übereinstimmung oder der Nichtübereinstimmung und des Widerstreits zwischen irgendwelchen von unseren Ideen «. 48 Locke unterscheidet hierbei zwischen drei Erkenntnisgraden: intuitive, demonstrative und sensitive Erkenntnis. >Intuitive Erkenntnis« nennt er den

$44 \quad$ E V 30; vgl. Lakebrink (1997, S. 704). Ellsiepen weist darauf hin, dass Spinozas Formulierung sub specie aeternitatis auch im Zusammenhang mit der Erkenntnisgattung der ratio verwendet wird; für die scientia intuitiva habe sie aber besonderes Gewicht, weil es hier »nicht nur um die Übereinstimmung von Begriffen mit der göttlichen Notwendigkeit der Naturordnung, sondern um die mentale Repräsentation jener Ewigkeit selbst« gehe (Ellsiepen 2006a, S. 148).

45 Förster (2011, S. 108).

46 Locke, 1.1.2 - Aus dem Essay wird wie folgt zitiert: erste Ziffer für das Buch (1-4), zweite Ziffer für die Nummer des Kapitels, dritte Ziffer für die Nummer des Abschnitts.

47 Vgl. Kobusch (1976, S. 530); Specht (1989, S. 123); Thiel (2006, S. 96 f., Fn. 5).

$48 \quad$ 4.1.2. 
Fall, in dem unser Geist die Übereinstimmung oder Nichtübereinstimmung der Ideen »unmittelbar durch sie selbst wahrnimmt, ohne Vermittlung einer dritten « ${ }^{49}$ Als Beispiele für solche intuitiven Erkenntnisse führt Locke an, »dass weiß nicht schwarz ist, dass ein Kreis kein Dreieck, dass drei mehr als zwei und gleich eins plus zwei ist «.50 Diese Erkenntnisse drängen sich Locke zufolge dem menschlichen Geist regelrecht auf, d.h. sie lassen »keinerlei Spielraum für ein Schwanken, Zweifeln oder Prüfen«, und »auf dieser Intuition beruht die ganze Gewissheit und Augenscheinlichkeit unseres gesamten Wissens « ${ }^{51}$ Wie bei Descartes und Spinoza ist die Intuition also auch bei Locke durch Unmittelbarkeit und Gewissheit gekennzeichnet.

Demonstrative Erkenntnis kommt dann zum Zug, wenn die intuitive Erkenntnis nicht ausreicht, da die Übereinstimmung oder Nichtübereinstimmung zweier Ideen nicht unmittelbar und zweifelsfrei einsichtig ist. Dies ist etwa bei schwierigeren Gleichungen oder anderen Beweisgängen der Fall. Hier müssen wir vermittelnde Ideen einsetzen um herauszufinden, ob die Ausgangsideen miteinander übereinstimmen oder nicht. Zum Beispiel können wir, so Locke, nicht im oben genannten Sinn intuitiv erkennen, ob die drei Winkel eines Dreiecks zwei rechten Winkeln entsprechen. Erst durch die Ermittlung anderer Winkel im Rahmen eines geometrischen Beweises können wir dies feststellen. ${ }^{52}$ Lockes demonstrative Erkenntnis entspricht somit dem Cartesischen Erkenntnisweg der Deduktion. Die Kette von Teilschritten zwischen den zu überprüfenden Ideen kann beliebig verlängert werden. Doch selbst wenn diese Deduktion fehlerfrei durchgeführt wird, fehlt der demonstrativen Erkenntnis die Klarheit der intuitiven Erkenntnis. Locke vergleicht eine solche lange Beweiskette anschaulich mit »einem Gesicht, das durch eine Reihe von Spiegeln immer wieder reflektiert wird «, wobei mit jeder Widerspiegelung das Gesicht diffuser und dunkler wird $«{ }^{53}$ Die demonstrative Erkenntnis führt, falls die entsprechende Demonstration bzw. Deduktion fehlerfrei durchgeführt wird, ebenso zuverlässig zu wahren Ideen wie die intuitive. Jedoch ist die Gewissheit bei der intuitiven Erkenntnis unmittelbar, d.h. es gibt keinen Spielraum dafür, die Zuverlässigkeit dieser Erkenntnis in Frage zu stellen. In diesem Sinn gilt die Höherrangigkeit der intuitiven Erkenntnis, wie für Descartes und Spinoza, auch für Locke.

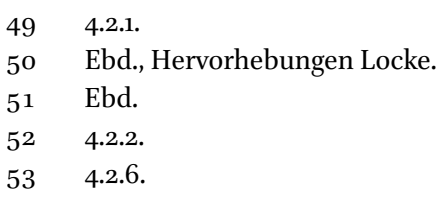


Eine weitere Übernahme von Descartes ist Lockes Verschränkung der intuitiven mit der demonstrativen Erkenntnis. Locke betont, genau wie Descartes, dass bei jedem demonstrativen bzw. deduktiven Schritt eine intuitive Erkenntnis bezüglich der Übereinstimmung oder Nichtübereinstimmung zur nächsten vermittelnden Idee gegeben sein muss. ${ }^{54}$ Lockes Intuitionskonzeption unterscheidet sich jedoch von den axiomatischen Konzeptionen Descartes' und Spinozas dadurch, dass die Übereinstimmung oder Nichtübereinstimmung unbegrenzt vieler Ideen auf intuitive Weise erkannt werden kann. Es gibt bei Locke keine selbstevidenten Axiome, aus denen alles Übrige abgeleitet werden kann. Die Selbstevidenz im Sinne der Lockeschen Intuition ergibt sich vielmehr an jeder beliebigen Stelle, an der die Übereinstimmung oder Nichtübereinstimmung zweier Ideen miteinander intuitiv klar wird. ${ }^{55}$

Den dritten Grad der Erkenntnis bezeichnet Locke als >sensitive Erkenntnisく. Während sich intuitive und demonstrative Erkenntnis auf allgemeine Wahrheiten beziehen, steht die sensitive Erkenntnis im Zusammenhang mit unserer Wahrnehmung vom »Einzeldasein endlicher Wesen außer uns « ${ }^{56} \mathrm{Im}$ Gegensatz zu den Rationalisten Descartes und Spinoza hält Locke sichere sensitive Erkenntnis für möglich. Allerdings räumt er ein, dass die sensitive Erkenntnis nicht dieselbe Gewissheit wie die demonstrative und die intuitive Erkenntnis aufweist. ${ }^{57}$

Im Zusammenhang mit der Unterscheidung zwischen intuitiver und sensitiver Erkenntnis zeichnen sich Probleme ab. Nehmen wir den Empiristen Locke beim Wort, müsste er Intuition strikt auf den Bereich der Sinneswahrnehmung beschränken. Wenn nämlich der Geist nichts weiter tun kann als das vorhandene Sinnesmaterial zu ordnen, vergleichen, kombinieren, etc., dann bleibt zwischen sensitiver und demonstrativer Erkenntnis keinerlei Raum für eine intuitive, nicht-sensitive Erkenntnisart; denn wenn Unmittelbarkeit besteht, ist noch keine Tätigkeit des Geistes vorhanden, und wenn letztere vorhanden ist, besteht keine Unmittelbarkeit mehr. Eine weitere Schwierigkeit in Bezug auf die sensitive Erkenntnis betrifft die Diskrepanz zu Lockes Erkenntnisdefinition als Wahrnehmung der Übereinstimmung zwischen Ideen. Während bei intuitiver und demonstrativer Erkenntnis die

\footnotetext{
54 Vgl. 4.2.7.

55 Es muss präzisiert werden, dass Locke hier im engeren Sinne axiomatische Systeme, wie sie die Folge der Cartesischen Konzeption sein konnten - wie allenfalls dasjenige Spinozas - angreift und nicht die Grundaussagen der Regulae; denn hier sagt Descartes nicht explizit, dass sich alle Erkenntnis auf einige wenige Axiome zurückführen lasse.

$56 \quad 4.2 .14$

57 Vgl. 4.2.14.
} 
Übereinstimmung zwischen Ideen geprüft wird, scheint dies bei der Sensation (d.h. dem Prozess der sensitiven Erkenntnis) nicht der Fall zu sein. ${ }^{58}$ Locke kann keinen Weg angeben, und es ist wohl auch kein Weg möglich anzugeben, wie die Existenz einzelner Dinge der Außenwelt aus dem Vergleich von Ideen hergeleitet werden kann. ${ }^{59}$ Damit müsste Locke entweder seine allgemeine Erkenntnisdefinition verändern oder aber die Ansicht aufgeben, dass es sich bei sensitiver Erkenntnis um Erkenntnis in dem von ihm angegebenen Sinn handelt.

Zusammenfassend zeigt sich, dass der Empirismus bei genauer Betrachtung keinen Spielraum für eine intuitive Erkenntnis zwischen der sensitiven und der demonstrativen Erkenntnis lässt. Mit dieser Einsicht ist der Weg für Immanuel Kant geebnet, der die Intuition in die Schranken des Empirischen verweist.

\subsection{Kant: Intellektuelle Anschauung und anschauender Verstand}

Immanuel Kants Transzendentalismus markiert einen bedeutenden Wendepunkt in der abendländischen Philosophiegeschichte, der auch das Verständnis von >Intuition< betrifft. Der allgemeine Wendepunkt wird - in Anlehnung an einen von Kant selbst vorgenommenen Vergleich in der Kritik der reinen Vernunft (KrV, 1781/1787) - oft als >kopernikanische Wende< bezeichnet. Wie Kopernikus gezeigt hatte, dass die Erde nicht im Mittelpunkt steht und die Gestirne um sie kreisen, sondern umgekehrt, so will Kant in Bezug auf die Erkenntnistheorie neu davon ausgehen, dass sich die Erkenntnis nicht nach dem Erkenntnisgegenstand, sondern umgekehrt der Gegenstand nach der Erkenntnis richtet. ${ }^{60}$ Aufgrund der Unverfügbarkeit des $>$ Dings an sich $<$ wandelt sich die Rolle der Philosophie grundlegend. Es kann nicht mehr darum gehen, über das Wesen der Dinge zu spekulieren; vielmehr muss die Philosophie zuerst die Bedingungen der Möglichkeit unserer Erkenntnis untersuchen.

Im Anschluss an die Kopernikanische Wende stellt sich die Frage neu, welche Rolle Sinnlichkeit und Verstand im Erkenntnisprozess einnehmen; denn nun kann weder davon ausgegangen werden, dass die Sinnlichkeit dem Verstand Zugang zum Wesen der Dinge verschafft, noch dass der Verstand allein zur Erkenntnis dieses Wesens fähig ist. Kants Antwort auf dieses Problem lautet, dass zwischen Sinnlichkeit und Verstand eine scharfe Trennlinie gezogen und eine komplementäre Rollenzuweisung in Bezug auf die

58 Vgl. 4.3.2.

59 Für einen ähnlichen Kritikpunkt siehe Lowe (1995, S. 174).

6o Vgl. KrV B XVI-XVIII. 
Erkenntnis vorgenommen werden muss. Dabei bestimmt der Verstand den Rahmen, in dem sich Erkenntnis ereignen kann (d.h., was uns als Erscheinung zugänglich wird), während die Sinnlichkeit das Material liefert, welches im Erkenntnisrahmen aufgenommen und entsprechend bearbeitet wird. Dieses $\mathrm{Zu}-$ sammenwirken von Sinnlichkeit und Verstand mündet in Kants berühmtem Diktum »Gedanken ohne Inhalt sind leer, Anschauungen ohne Begriffe sind blind ${ }^{6}{ }^{61}$

Wie positioniert sich Kant in Bezug auf die Frage der Intuition? Zunächst ist $\mathrm{zu}$ beachten, dass Kant den Begriff >Anschauung< verwendet, also die deutsche Übersetzung des Wortes >Intuition ${ }^{62}$ Nachdem Kant Sinnlichkeit und Verstand scharf voneinander getrennt hat, ordnet er die Anschauung der Sinnlichkeit zu. Intuition, die über bloße Wahrnehmung hinausgeht, ist Kant zufolge dem menschlichen Verstand nicht gegeben. Somit reduziert Kant die Intuition auf den Beitrag der Sinneswahrnehmung zu einem durch und durch diskursiv (bzw. bei Descartes: >deduktiv<, bei Locke: >demonstrativ<) verfassten Erkenntnisprozess.

Mit dieser Eingrenzung des Intuitionsbegriffs auf die Sinnlichkeit verneint Kant im Hinblick auf den Menschen das Bestehen einer höheren Erkenntnisgattung, die das Wesen der Dinge hinter den Erscheinungen ausmachen könnte. Dies richtet sich auch gegen die oben dargestellte Intuitionskonzeption Spinozas: ${ }^{63}$

Wenn die Klagen:Wir sehen das Innere der Dinge gar nicht ein, so viel bedeuten sollen, als, wir begreifen nicht durch den reinen Verstand, was die Dinge, die uns erscheinen an sich sein mögen; so sind sie ganz unbillig und unvernünftig; denn sie wollen, dass man ohne Sinne doch Dinge erkennen, mithin anschauen könne, folglich dass wir ein von dem menschlichen [...] gänzlich unterschiedenes Erkenntnisvermögen haben, also nicht Menschen, sondern Wesen sein sollen, von denen wir selbst nicht angeben können, ob sie einmal möglich, vielweniger, wie sie beschaffen sein. ${ }^{64}$

War es bei Spinoza noch möglich, dass wir via scientia intuitiva auch als endliche Wesen an der göttlichen Erkenntnisart Anteil nehmen, weist Kant diese Vorstellung als irrig zurück: Wir sind keine Götter und können daher auch nicht intuitiv erkennen. Dieser Kritik an rationalistischen und idealistischen Positionen sind wir bereits bei Locke begegnet. Wie dort ersichtlich wurde,

\footnotetext{
$61 \quad \mathrm{KrV} B 75$.

62 In den englischsprachigen Kant-Ausgaben und Sekundärliteratur wird >Anschauung< zu intuition zurückübersetzt.

63 Vgl. Förster (2011, S. 254f.).

$64 \quad \mathrm{KrV}$ B $333-334$.
} 
bleibt Locke im Grunde kein Spielraum für die Intuition als eigenständige Erkenntnisgattung zwischen sensitiver und demonstrativer Erkenntnis. Kant führt diese empiristische Einsicht zu ihrer Konsequenz, indem er die Möglichkeit einer nichtsinnlichen Intuition explizit zurückweist.

Im Folgenden soll zunächst genauer aufgezeigt werden, was Kant unter Intuition (d.h. unter der eigentlichen Intuition im vorkantischen Sinne) versteht und warum er diese als für das menschliche Erkenntnissubjekt nicht verfügbar ansieht. Anschließend kann die Frage angegangen werden, inwiefern auch nach Kant noch Spielraum für Intuition als eigenständige Erkenntnisgattung bleiben kann.

Aus der Trennung von Rezeptivität und Spontaneität ergeben sich zwei Quellen der Erkenntnis, die Kant zufolge menschenunmöglich sind: Erstens die intellektuelle Anschauung, da Anschauung immer rezeptiv ist, zweitens der intuitive Verstand, da der Verstand auf Diskursivität festgelegt ist. ${ }^{65}$ Kant erörtert an verschiedenen Stellen, vor allem in der $\mathrm{KrV}$, aber auch in der Kritik der Urteilskraft (KdU), was unter >intellektueller Anschauung b bzw. >anschauendem Verstand zu verstehen wäre.

Betrachten wir zunächst Kants Begriffsbestimmung der sintellektuellen Anschauung in Zusammenhang mit den philosophiegeschichtlich verankerten und in Kants erkenntnistheoretischem Rahmen ebenfalls bedeutsamen Begriffen Phaenomenon und Noumenon. Unter Phaenomena versteht Kant »Erscheinungen, so fern sie als Gegenstände nach der Einheit der Kategorien gedacht werden « ${ }^{66}$ Phaenomena bezeichnen Erscheinungen, also die Art, in der uns Erkenntnisobjekte durch unsere Anschauungs- und Denkformen gegeben sind. Für Noumena erwägt Kant zunächst zwei Begriffsbestimmungen:

Wenn wir unter Noumenon ein Ding verstehen, so fern es nicht Objekt
unserer sinnlichen Anschauung ist, indem wir von unserer Anschau-
ungsart desselben abstrahieren; so ist dieses ein Noumenon im negativen Ver-
stande. Verstehen wir aber darunter ein Objekt einer nichtsinnlich en
Anschauung, so nehmen wir eine besondere Anschauungsart an, nämlich die
intellektuelle, die aber nicht die unsrige ist, von welcher wir auch die Möglichkeit
nicht einsehen können, und das wäre das Noumenon in positiver Bedeutung.

Zum einen können Noumena also negativ dasjenige bezeichnen, welches uns nicht in der Anschauung gegeben ist und wir somit nicht erkennen können, jedoch als Ursache der uns zugänglichen Erscheinung postuliert werden muss.

\footnotetext{
65 Vgl. Förster (2011, S. 154).

$66 \mathrm{KrV}$ A 249.

$67 \quad \mathrm{KrVB} 307$.
} 
Zum anderen können Noumena Objekte einer intellektuellen Anschauung sein. Kant weist das Bestehen dieser Art von Anschauung und damit das traditionelle Noumenon-Verständnis »in positiver Bedeutung « zurück, welches etwa in Spinozas Erkenntnislehre zentral war. Das Kapitel Phaenomena und Noumena in der KrV schließt mit der entsprechenden Bemerkung: »was also von uns Noumenon genannt wird, muss als ein solches nur in negativer Bedeutung verstanden werden. $\ll^{68}$

Neben der Zurückweisung der intellektuellen Anschauung anhand der Negierung deren Objekts (des Noumenon im positiven Sinn) nimmt Kant die Zurückweisung auch anhand genauerer Überlegungen zur Begriffsbestimmung vor. Dies zeigt sich in seiner Diskussion des Beispiels >Selbstbewusstsein<:

Das Bewusstsein seiner selbst (Apperzeption) ist die einfache Vorstellung des Ich, und, wenn dadurch allein alles Mannigfaltige im Subjekt selbsttätig gegeben wäre, so würde die innere Anschauung intellektuell sein. ${ }^{69}$

Kant zufolge können einfache Vorstellungen, wie es die Anschauungen sind, nicht selbsttätig die Begriffe hervorbringen, unter welche die Objekte der Anschauungen zu subsumieren sind. Beispielsweise können wir aus der Anschauung des einzelnen Junggesellen Kant allein nicht ableiten, welche anderen Männer in diese Kategorie fallen, denn aus der einfachen Anschauung erschließt sich uns nicht, welche Begriffskategorie gebildet werden soll. Der Begriff zieht also selbsttätig (= spontan-aktiv) eine Grenze um die durch die Anschauung gegebenen Gegenstände, die unter ihn fallen sollen (vgl. die Gemeinbegriffe bei Spinoza). Die rezeptiv-passive Anschauung kann diese Bestimmung nicht allein hervorrufen, weshalb der diskursive Verstand die bestimmenden Begriffe hinzufügen muss. So kann im genannten Beispiel der Gehalt des Begriffs >Ich ^ nicht selbsttätig aus einer ichbezogenen Sinneswahrnehmung erschlossen werden. Vielmehr bleibt es angesichts der blinden Anschauung der Willkür der diskursiven Begriffsbildung überlassen, welche Anschauungen unter dem Begriff $>$ Ich $<$ subsumiert werden.

Auch das Gegenstück zur intellektuellen Anschauung, den anschauenden Verstand, bestimmt Kant zunächst genauer, um diesen dem Menschen ebenfalls abzusprechen. Während der diskursive Verstand »vom AnalytischAllgemein en (von Begriffen) zum Besondern (der gegebenen empirischen Anschauung) gehen muss«, geht der intuitive Verstand »vom SynthetischAllgemeinen (der Anschauung eines Ganzen als eines solchen) zum

$68 \mathrm{KrVB} 309$.

$69 \quad \mathrm{KrV} B 68$. 
Besondern [...], d. i. vom Ganzen zu den Theilen $\ll^{70}$ Ein solcher intuitiver Verstand würde im Gegensatz zum diskursiven Verstand »die Möglichkeit der Theile (ihrer Beschaffenheit und Verbindung nach) als vom Ganzen abhängend vorstellen«, dergestalt, dass »das Ganze den Grund der Möglichkeit der Verknüpfung der Theile « vorgibt. ${ }^{71}$ Mit unserem diskursiven Verstand hingegen ist es unmöglich, »aus der Natur selbst hergenommene Erklärungsgründe für Zweckverbindungen zu schöpfen«, da sich unsere Erkenntnis immer im Rahmen der a priori bestehenden Anschauungsform der (Natur-) Kausalität bewegen muss. ${ }^{72}$ Nur wenn wir Anteil haben könnten an »einem ursprünglichen Verstande als Weltursache«, wie Kant den intuitiven Verstand auch beschreibt, wäre uns mehr möglich. ${ }^{73}$ Kant weist also sowohl die Möglichkeit einer intellektuellen Anschauung als auch diejenige eines intuitiven Verstandes zurück, welches die beiden Weisen sind, auf die sich Intuition als Erkenntnisart jenseits des rein Empirischen konstituieren könnte.

Eckart Förster vertritt in seiner Abhandlung Die 25Jahre der Philosophie die These, dass Kants Zurückweisung der Intuition nicht berechtigt ist. ${ }^{74}$ Förster zeigt auf, inwiefern der in der $\mathrm{KrV}$ postulierte strenge Dualismus von sinnlicher Anschauung und diskursivem Verstand und die damit verbundene Ablehnung der intuitiven Erkenntnis bereits von Philosophen des deutschen Idealismus revidiert wurde. Diese Rehabilitierung der nicht rein empirischen Intuition gelingt so früh, weil Kants Zurückweisung der intuitiven Erkenntnis eine alleinstehende Begründung fehlte. Dass dem Menschen nur diskursive Erkenntnis möglich sei, bleibt letztlich eine Behauptung, die nicht über die Vorgaben des Transzendentalismus hinaus begründet werden kann. ${ }^{75}$ Einen Anstoß für die Rehabilitierung der einen Art von intuitiven Erkenntnis, des intuitiven Verstandes, gibt Kant in der KdU bereits selber. Kant stellt hier den intuitiven Verstand so dar, dass nicht mehr klar ist, warum diese Art Verstand dem Menschen vorenthalten sein sollte. In der bereits besprochenen Passage schreibt Kant, der intuitive Verstand gehe »vom Syn th et is ch-Allg eme in en (der Anschauung eines Ganzen als eines solchen) zum Besondern [...], d. i. vom Ganzen zu den Theilen $\ll^{76}$ Dieser Gang vom Synthetisch-Allgemeinen zum Besonderen ist prinzipiell auch für einen Verstand möglich, der dieses Besondere nicht selber hervorbringt, also für einen endlichen, nicht-göttlichen

\begin{tabular}{ll}
\hline 70 & Kant, AA V 407. \\
71 & Ebd. \\
72 & AA V 410. \\
73 & Ebd. \\
74 & Vgl. Förster (2011). \\
75 & Vgl. Förster (2011, S. 256). \\
76 & AA V 407.
\end{tabular}


Verstand. ${ }^{77}$ Einzig der Umstand, dass Kant dem Menschen partout nur diskursives Erkenntnisvermögen zugestehen will, spricht dagegen, womit seine Argumentation zirkulär bleibt.

Auch die Möglichkeit der intellektuellen Anschauung wird bald nach Kant wieder diskutiert. Die Intuition nimmt nicht nur in den von Förster erwähnten Arbeiten des deutschen Idealismus, sondern später auch im philosophischen Werk Arthur Schopenhauers eine zentrale Rolle ein. Schopenhauer kritisiert Kants Transzendentalismus und mit diesem die Vorstellung, wonach die Anschauung rein empirisch sei. Diese, so Schopenhauer, vernachlässige die zentrale Unterscheidung zwischen Anschauung und Empfindung (verstanden als bloß physiologische Sinnesreizung). Mit der Losung »Alle Anschauung ist eine intellektuale « ${ }^{78}$ führt Schopenhauer die Intuition als Erkenntnisvermögen zwischen Empfindung und Vernunft ein und verneint somit Kants Auffassung der Intuition als vollständig rezeptiv-empirisch.

Kants begriffliche Aufspaltung der Intuition in sintellektuelle Anschauung und sintuitiver Verstand< ermöglicht somit eine Re-Etablierung der Intuition auf den beiden voneinander zu unterscheidenden Wegen. Hier soll im Folgenden auf den Weg des intuitiven Verstandes fokussiert werden, da sich die Diskussion zur intellektuellen Anschauung vom Thema der Intuition im engeren Sinn entfernen würde: Hier ginge es vor allem auch darum zu untersuchen, inwiefern die Perzeption bereits von kognitiven Elementen durchdrungen ist. Dies ist ein interessantes und aktuelles Thema, zu dessen Bearbeitung auch kognitionswissenschaftliche und psychologische Befunde einen entscheidenden Beitrag leisten. ${ }^{79}$ Darauf wird im Folgenden aber nicht eingegangen. Stattdessen soll zunächst die Möglichkeit des intuitiven Verstands anhand von Goethes Wiederaufnahme der Spinozistischen Intuitionskonzeption erörtert werden.

\subsection{Goethe: Die Möglichkeit des intuitiven Verstandes}

Wer zu vertieften Einsichten in Bezug auf die Natur gelangen möchte, könnte sich in besonderem Maße für die Möglichkeit eines intuitiven Verstandes interessieren, wie am Beispiel von Johann Wolfgang von Goethes (1749-1832) Naturforschung deutlich wird. Der Grund für den potentiell privilegierten

\footnotetext{
77 Vgl. Förster, (2011, S. 153); Haag/Wild, (2013, S. 13).

78 Schopenhauer, VS, §1, S. 647; siehe auch WWV, §4, S. 41.

79 Siehe etwa die Überlegungen und Untersuchungen zu visueller Abduktion (vgl. Magnani 2001, S. 97-115).
} 
Zugang des intuitiven Verstandes zur Natur liegt darin, dass der intuitive Verstand das Wesen der Einzeldinge erfassen, also zu Wesensbegriffen vordringen kann. Dem diskursiven Verstand, und somit auch Kants Erkenntnistheorie, bleibt dieser Zugang hingegen verwehrt. Was hier genau fehlt, lässt sich anhand eines der Beispiele Kants darstellen, welches er in der Schrift Logik(180o) festhält:

Ich sehe z. B. eine Fichte, eine Weide und eine Linde. Indem ich diese Gegenstände zuvörderst untereinander vergleiche, bemerke ich, daß sie von einander verschieden sind in Ansehung des Stammes, der Äste, der Blätter u. dgl. m.; nun reflectire ich aber hiernächst nur auf das, was sie unter sich gemein haben, den Stamm, die Äste, die Blätter selbst und abstrahire von der Größe, der Figur derselben u.s.w.; so bekomme ich einen Begriff vom Baume. ${ }^{80}$

Bei der diskursiven Begriffsbildung prägt der Verstand der Anschauung die Begriffe willkürlich auf, während der >Baum an sich eine Leerstelle bleibt. Anders gesagt: Wir hätten auch andere Gemeinsamkeiten auswählen und von anderen Unterschieden abstrahieren können und wären dadurch trotz derselben Anschauungen möglicherweise zu einem ganz anderen Begriff gelangt, sodass es in unserer Naturbeschreibung den Begriff >Baum< gar nicht gäbe. Die Teile (Stamm, Blätter, Äste, usw.) und das Ganze (Baum) sind in dieser Sichtweise nur unidirektional miteinander verknüpft: Die Teile (bzw. welche Gemeinsamkeiten ich für relevant erkläre und von welchen Verschiedenheiten ich abstrahiere) bedingen in der diskursiven Begriffsbildung das Ganze (>Baum<), aber umgekehrt bedingt das Ganze nicht die Teile.

Nun ist dies insbesondere in Bezug auf lebendige Organismen eine unbefriedigende Weise der Begriffsbildung, denn in einem Organismus wirken Ganzes und Teile dergestalt zusammen, dass die Teile nicht nur das Ganze, sondern das Ganze auch seine Teile bedingt. Jede Naturforscherin möchte sagen können, dass ein Organismus sich von seiner Umwelt aufgrund eines immanenten Zusammenhaltens und v.a. Zusammenwirkens seiner Teile abgrenzt, nicht aufgrund einer willkürlichen Begriffsbestimmung. ${ }^{81}$ Hier kommt der intuitive Verstand ins Spiel: Er kann »vom Synthetisch-Allgemeinen (der Anschauung eines Ganzen als eines solchen) zum Besondern« übergehen, also Wesensbegriffe erfassen.

\section{8o $\quad$ AA IX 94 f.}

81 Vgl. Wild (2012, S. 3) - Bei lebenden Organismen wird dieser Wunsch der Naturforscherin besonders deutlich nachvollziehbar, er kann aber auch auf nichtorganische Entitäten, die ein Funktionsganzes sind, ausgedehnt werden, wie sich etwa in Goethes Schriften zur Morphologie zeigt: So möchte auch der Geologe das Jura-Gebirge als Funktionsganzes sehen, das durch bestimmte inhärente Merkmale, die in einer spezifischen Weise zusammenwirken, definiert wird. 
Die Aussicht eines derartigen Zugangs zum Wesen der Einzeldinge motiviert auch Goethes Beschäftigung mit der scientia intuitiva im Rahmen seiner naturwissenschaftlichen Studien. Mit guter Kenntnis von sowohl Kants als auch Spinozas Arbeiten zur Intuition veröffentlicht Goethe 1790 den Versuch, die Metamorphose der Pflanzen zu erklären. Hier, wie auch später in der Farbenlehre, kommt Goethes Programm einer naturwissenschaftlichen intuitiven Erkenntnis zum Tragen.

Spinoza ist eine wichtige Quelle für Goethe, da jener die scientia intuitiva als Königsweg zur Erkenntnis betrachtet. Mit Spinoza lässt sich die Kritik der Naturforscherin an Kants einseitig diskursiver Begriffsbestimmung auf den Punkt bringen: Diese Art willkürlicher Begriffe sagen nur etwas über die Vorstellung des Menschen aus, jedoch nichts über die Dinge, wie sie an sich in der Natur sind; sie sind in Spinozas Terminologie nur auf opinio und imaginatio bezogen und liefern somit keine adäquate Erkenntnis. Die Kantische Replik ist eine Bestätigung und Kritik dieses Punktes zugleich: Kant würde bestätigen, dass er sich von der Idee einer adäquaten Erkenntnis verabschiedet hat, denn diese ist Kant zufolge schlicht falsch: Wir haben keinen Zugang zu den Dingen an sich. Was für Kant eine »Grille« ist, ist für Spinoza eine »höhere Wahrheitsnorm «. 82

Spinoza hat uns jedoch, wie Goethe feststellt, noch keinen Weg aufgezeigt, wie intuitive Erkenntnis in Bezug auf die Natur möglich werden kann. Nicht zufällig stammen Spinozas Beispiele meist aus dem Bereich der Mathematik. Auch zeigen sich weitere Probleme, die im Zusammenhang mit dem metaphysischen Grundgerüst von Spinozas Erkenntnistheorie stehen. Daher strebt Goethe nun eine Verbindung zwischen diskursiver und intuitiver Erkenntnis an. Er ist als Naturforscher kein Geist-Monist wie Spinoza; wir müssen die Anschauungen in Bezug auf die Natur zuerst haben und danach von diesem diskursiv »aufgesammelten « Einzelnen zum Allgemeinen gehen, also zumindest zu Beginn den diskursiv-analytischen Weg beschreiten. ${ }^{83}$ Daraus kann aber, wie Goethe hofft, eine intuitive Erkenntnis das den einzelnen Anschauungen zugrundeliegende gemeinsame Wesen offenbaren. Dieser Prozess soll im Folgenden anhand von Goethes Arbeiten zur Pflanzenmetamorphose illustriert werden. ${ }^{84}$

82 Förster (2011, S. 255).

83 Vgl. Förster (2011, S. 258); Haag/Wild (2013, S. 19).

84 Eine ausführlichere Darstellung dieser Arbeiten Goethes findet sich im 11. Kapitel von Försters Die 25 Jahre der Philosophie (Förster 2011, S. 253-276). Förster stellt dort Goethes Farbenlehre in den Kontext des intuitiven Verstandes. Für die folgende Darstellung wurde nur die Pflanzenlehre gewählt, da die Farbenlehre nicht eine gleichermaßen direkte Anwendung des intuitiven Verstandes darstellt (vgl. Förster, 2013, S. 353f.). 
Wie am Beispiel des Baums gezeigt wurde, geht Kant davon aus, dass der Begriff $>$ Pflanze< allein durch die reflektierende Urteilskraft zustande kommt, d.h. diskursiv-analytisch, und nicht durch die bestimmende Urteilskraft. ${ }^{85}$ Goethe will, in Anlehnung an Spinoza, über diese bloßen modi imaginandi hinauskommen und die Pflanzenbegriffe als Wesensbegriffe, d.h. intuitiv, erfassen können. In seinem Versuch, die Metamorphose der Pflanzen zu erklären (1790) stellt er zu diesem Zweck zunächst viele Einzelbeobachtungen an, welche die einjährigen Blütenpflanzen in verschiedenen Stadien ihres Lebenszyklus betreffen. Anhand dieser Einzelbeobachtungen erkennt Goethe ein grundlegendes Muster, das dem ganzen Lebenszyklus der Pflanzen wesentlich zugrunde liegt, ohne sich direkt in einer oder mehreren Anschauungen zu zeigen:

Vom Samen bis zu der höchsten Entwicklung des Stengelblattes bemerkten wir zuerst eine Ausdehnung, darauf sahen wir durch eine Zusammenziehung den Kelch entstehen, die Blumenblätter durch eine Ausdehnung, die Geschlechtsteile abermals durch eine Zusammenziehung; und wir werden nun bald die größte Ausdehnung in der Frucht und die größte Konzentration in dem Samen gewahr werden. In diesen sechs Schritten vollendet die Natur unaufhaltsam das ewige Werk der Fortpflanzung der Vegetabilien durch zwei Geschlechter. ${ }^{86}$

Das Muster ist also der stetige Wechsel zwischen Ausdehnung und $\mathrm{Zu}$ sammenziehung. Damit ist nicht eine äußerlich immer gleich beobachtbare Ausdehnung bzw. Zusammenziehung gemeint, sondern dieser Wechsel ist ein Prinzip, das sich in Bezug auf verschiedene Teile der Pflanze und in konkreten Bewegungen äußert. ${ }^{87}$ Nach Goethe sind wir mit dieser Erkenntnis zu einem vorgestellten Organ vorgedrungen, welches er »das ideelle Blat« nennt; auch der von ihm verwendete Begriff >Urpflanze< passt in diesen Zusammenhang. Hierbei handelt es sich um einen Wesensbegriff, was dadurch ersichtlich wird, dass der Beobachter auch in umgekehrter Richtung vorgehen und aus der Urpflanze verschiedene Pflanzenstadien bzw. Pflanzenarten und -formen rekonstruieren kann, die es weiter geben könnte. Der intuitive Verstand, der vom synthetisch-Allgemeinen zum Besonderen geht, ist hier am Werk. ${ }^{88}$

Goethe beschreibt sein Vorgehen bei naturwissenschaftlichen Studien in der Schrift Der Versuch als Vermittler zwischen Objekt und Subjekt am Beispiel der Optik:

85 Vgl. Förster (2011, S. 272).

86 GHA 13 84f. - Goethe wird nach der Hamburger Ausgabe (GHA) zitiert.

87 Vgl. Förster (2011, S. 273).

88 Vgl. Förster (2011, S. 274 f.). 
Ich habe in den zwei ersten Stücken meiner optischen Beiträge eine solche Reihe von Versuchen aufzustellen gesucht, die zunächst aneinander grenzen und sich unmittelbar berühren, ja, wenn man sie alle genau kennt und übersieht, gleichsam nur einen Versuch ausmachen, nur eine Erfahrung unter den mannigfaltigsten Ansichten darstellen. Eine solche Erfahrung, die aus mehreren andern besteht, ist offenbar von einer höhern Art. Sie stellt die Formel vor, unter welcher unzählige einzelne Rechnungsexempel ausgedrückt werden. Auf solche Erfahrungen der höhern Art loszuarbeiten, halt ich für höchste Pflicht des Naturforschers, und dahin weist uns das Exempel der vorzüglichsten Männer, die in diesem Fache gearbeitet haben $[\ldots] .{ }^{89}$

Zunächst also macht Goethe viele einzelne Beobachtungen, die zeitlich und/ oder räumlich nahe beieinander liegen (hier: die vielen Stadien der einjährigen Blütenpflanze betreffend). Dies sind die einzelnen Anschauungen, die zuerst gesammelt und angeordnet werden. Der Beginn bei den einzelnen Anschauungen, und damit die Bewegung vom Besonderen zum Allgemeinen, stellt das diskursive Element in der Naturforschung dar. In einem nächsten Schritt, der den Wendepunkt vom diskursiven hin zum intuitiven Erkennen markiert, richtet der Naturforscher Goethe besonderes Augenmerk auf die Übergänge zwischen den einzelnen Anschauungen. Durch die Anordnung und Betrachtung der Übergänge wird plötzlich ein den einzelnen Anschauungen zugrundeliegender Wesensbegriff erkennbar, der sie hervorbringt. Der Wesensbegriff ist somit dynamisch, denn er schließt den Wandel der Anschauungen mit ein. Zugleich erweist er die Anschauungen als nicht zufällig zusammengefasst, sondern essentiell miteinander verbunden.

$\mathrm{Ab}$ hier ist der intuitive Verstand, passend zu Kants Beschreibung in der KdU, am Werk: Er arbeitet produktiv und in der entgegengesetzten Richtung: Vom gefundenen allgemein-synthetischen Wesensbegriff kann er zu einzelnen Anschauungen kommen. Dies sind im Beispiel der Pflanzenlehre die vielen weiteren hypothetischen Pflanzen oder Pflanzenstadien, die Goethe aus dem >ideellen Blat< bzw. der >Urpflanze< ableiten kann.

Diese Schilderungen zur Tätigkeit des intuitiven Verstandes sind zugegebenermaßen noch vage; vieles bleibt bei Goethe bloß angedeutet. Förster führt dessen Gedanken etwas weiter und stellt eine Regel auf. Im Zusammenhang mit Goethes Beispielen aus der Naturforschung haben wir es demnach mit drei Größen zu tun: a) Ursache, b) Produkt und c) durchgängige Tätigkeit. ${ }^{90}$ Angewandt auf das Pflanzenbeispiel laßen sich diese drei Teile wie folgt beschreiben:

89 Goethe, GHA 1318.

90 Vgl. Förster (2013, S. 362). 
a) der Wesensbegriff, den wir mit dem intuitiven Verstand erkennen, also das >ideelle Blat $<$ bzw. die >Urpflanze<;

b) die Anschauungen, die wir in der Versuchsreihe tätigen, also die einzelnen Pflanzen oder Entwicklungsstadien einer Pflanze;

c) die Übergänge zwischen diesen einzelnen Pflanzen oder Entwicklungsstadien.

Wenn uns zwei dieser Größen bekannt sind, lässt sich die dritte prinzipiell daraus ableiten:91

- Wesensbegriff und Anschauungen sind gegeben, Übergänge werden erschlossen: Haben wir einen experimentellen Film zu Ende gesehen und betrachten danach nochmals einzelne Szenen, so kann sich uns der zuvor rätselhafte Zusammenhang zwischen einzelnen Szenen erschließen, denn wir vermögen die Szenen nun in Bezug auf die gesamte Filmhandlung bzw. Grundidee einzuordnen.

- Wesensbegriff und Übergänge sind gegeben, Anschauungen werden erschlossen: Eine Grundidee für einen Spielfilm ist gegeben, ebenso der rote Faden der Handlung. Nun lassen sich daraus die einzelnen Szenen erarbeiten, also das Drehbuch schreiben. Von einem Film müssen mindestens die Grundidee sowie Anfang, Mitte und Ende bekannt sein. Dann lassen sich die einzelnen Szenen quasi handwerklich daraus konstruieren.

- Anschauungen und Übergänge sind gegeben, Wesensbegriff wird erschlossen: Hierzu führt Förster kein weiteres Beispiel an, weil genau dieser Fall auf die bereits dargestellten Naturstudien Goethes zutrifft. Hier sind die einzelnen Beobachtungen, welche die Versuchsreihe ausmachen, gegeben. Goethe hat in diskursiver Manier mit diesen angefangen, woraus sich unweigerlich die Übergänge ergeben. Aus diesen lässt sich schließlich das >ideelle Blat bzw. die >Urpflanze $<$ erkennen .

Der dritte Fall ist sicherlich der maßgebende, da die unbekannte Größe in der Regel der Wesensbegriff ist, insofern uns die Anschauungen vor Augen stehen und sich die Übergänge ergeben, wenn wir eine Versuchsreihe durchführen. Die beiden anderen Fälle sind trotzdem instruktiv, weil sie einen zentralen Punkt vor Augen führen: Sobald wir den Wesensbegriff erfasst haben, lässt sich das wahre Bedingungsverhältnis verfolgen: Dieses führt vom Wesensbegriff zu den einzelnen Anschauungen (und zu den Übergängen).

91 Vgl. Förster (2011, S. 261 f.) - Wichtig ist sich dessen bewusst zu bleiben, dass es sich um Veranschaulichungen und nicht mit der Naturforschung vergleichbare Fälle handelt. So ist etwa der Film ein Kunstprodukt. Ein genuines Beispiel wären hingegen die beschriebenen Pflanzenstudien Goethes. 


\subsection{Ausblick}

Zum Schluss dieses Kapitels sollen die für den Fortgang der Untersuchung zentralen Ergebnisse festgehalten werden, die sich aus der Darstellung und Diskussion der fünf neuzeitlichen Positionen zur Intuition ergeben haben.

Erstens kann abgeleitet werden, dass Unmittelbarkeit und Gewissheit zentrale Merkmale der Intuition sind. Das heißt: Wenn ein Subjekt eine Intuition im epistemischen Sinn (d.h. eine potentiell zu Erkenntnis führende Intuition) hat, erlebt es diese als unmittelbar und gewiss. Dies ließ sich bereits aus Descartes' und Spinozas Arbeiten ableiten, insbesondere aus deren Beispielen (z.B. >ich denke, ich existiereく, der Kaufmann). Da diese Beispiele wohl uns allen bekannte Erlebnisse ansprechen, die verbreitet als >Intuition< bezeichnet werden, soll die Annahme, dass Unmittelbarkeit und Gewissheit entscheidende Merkmale der Intuition sind, weiterverfolgt und präzisiert werden.

Zweitens muss festgestellt werden, dass die Frage, ob die Intuition eine epistemisch privilegierte Erkenntnisart ist, vorerst offen bleibt. Bei den rationalistischen Intuitionskonzeptionen Descartes' und Spinozas wird zwar postuliert, dass dem so sei; aber inwiefern dieser Anspruch unabhängig von einer schon geteilten rationalistischen Position bzw. apriorischen Intuitionsauffassung gelten darf, ist ungewiss. Mehr noch: Selbst Descartes war sich der Intuition - obwohl sie als gewiss erlebt wird - zunehmend weniger sicher. Die Intuitionsskepsis mündete in Kants Diktum, wonach die intellektuelle Intuition eine Grille sei.

Drittens jedoch haben wir mit Goethe gesehen, dass intellektuelle Intuition, bzw. ein anschauender Verstand, trotz der berechtigten Vorbehalte menschenmöglich sein könnte. Auch hier scheinen die Beispiele aus dem Bereich der Pflanzenstudien, verstärkt durch die hilfreichen Interpretationen Försters, für sich zu sprechen und prima facie mindestens so stark zu überzeugen, dass diese Möglichkeit für weitere Untersuchungen nicht ausgeschlossen werden darf. Doch bedeutet dies, dass wir mittels der Intuition zu Wesensbegriffen vordringen können? Hier stellt sich wieder die epistemologisch-skeptische Frage. Um Fragen wie diese anzugehen, ist es unabdingbar nun genauer $\mathrm{zu}$ untersuchen was vor sich geht, wenn wir Intuitionen haben und welche epistemologischen Konsequenzen daraus abgeleitet werden können. 